\title{
Metabolic Model for Autoregulation in the Circle of Willis
}

\author{
K. T. Moorhead, J. G. Chase, T. David, J. Arnold \\ Dept of Mechanical Engineering, University of Canterbury \\ Private Bag 4800, Christchurch, New Zealand
}

\begin{abstract}
The Circle of Willis (CoW) is a ring-like structure of blood vessels found at the base of the brain. Its main function is to distribute oxygen-rich arterial blood to the cerebral mass. In a previous study, a one-dimensional model of the CoW was created to simulate a series of possible clinical scenarios such as occlusions in afferent arteries, absent or string-like circulus vessels, or arterial infarctions. The model captured cerebral haemodynamic autoregulation by using a Proportional-IntegralDerivative (PID) controller to modify efferent artery resistances. Although some good results and correlations were achieved, the model was too simple to capture all the transient dynamics of autoregulation. Hence, a more physiologically accurate model has been created that additionally includes the oxygen dynamics that drive the autoregulatory response. Results very closely match accepted physiological response and limited clinical data. In addition, a set of boundary conditions and geometry is presented for which the autoregulated system cannot provide sufficient perfusion, representing a condition with increased risk of stroke and highlighting the importance of modelling the haemodynamics of the CoW. The system model created is computationally simple so it can be used to identify at-risk cerebral arterial geometries and conditions prior to surgery or other clinical procedures.
\end{abstract}

Keywords: Circle of Willis; Cerebral Haemodynamics; Computational Model; Autoregulation; PID controller 


\section{Introduction}

The Circle of Willis (CoW) is a ring-like structure of blood vessels found at the base of the brain. Its main function is to distribute oxygen-rich arterial blood to the cerebral mass. Although the brain comprises only approximately $2 \%$ of the total body mass, it demands approximately $20 \%$ of the body's oxygen supply. If the brain cells are starved of oxygen for more than a few minutes due to decreased flow and/or perfusion pressure, they become permanently damaged [1]. Hence, interstitial oxygen partial pressures are a major component in the regulation of cerebral blood supply to a tight range. Figure 1 shows a basic schematic of the Circle of Willis, labelling the major arteries considered in this study along with their abbreviations [2].

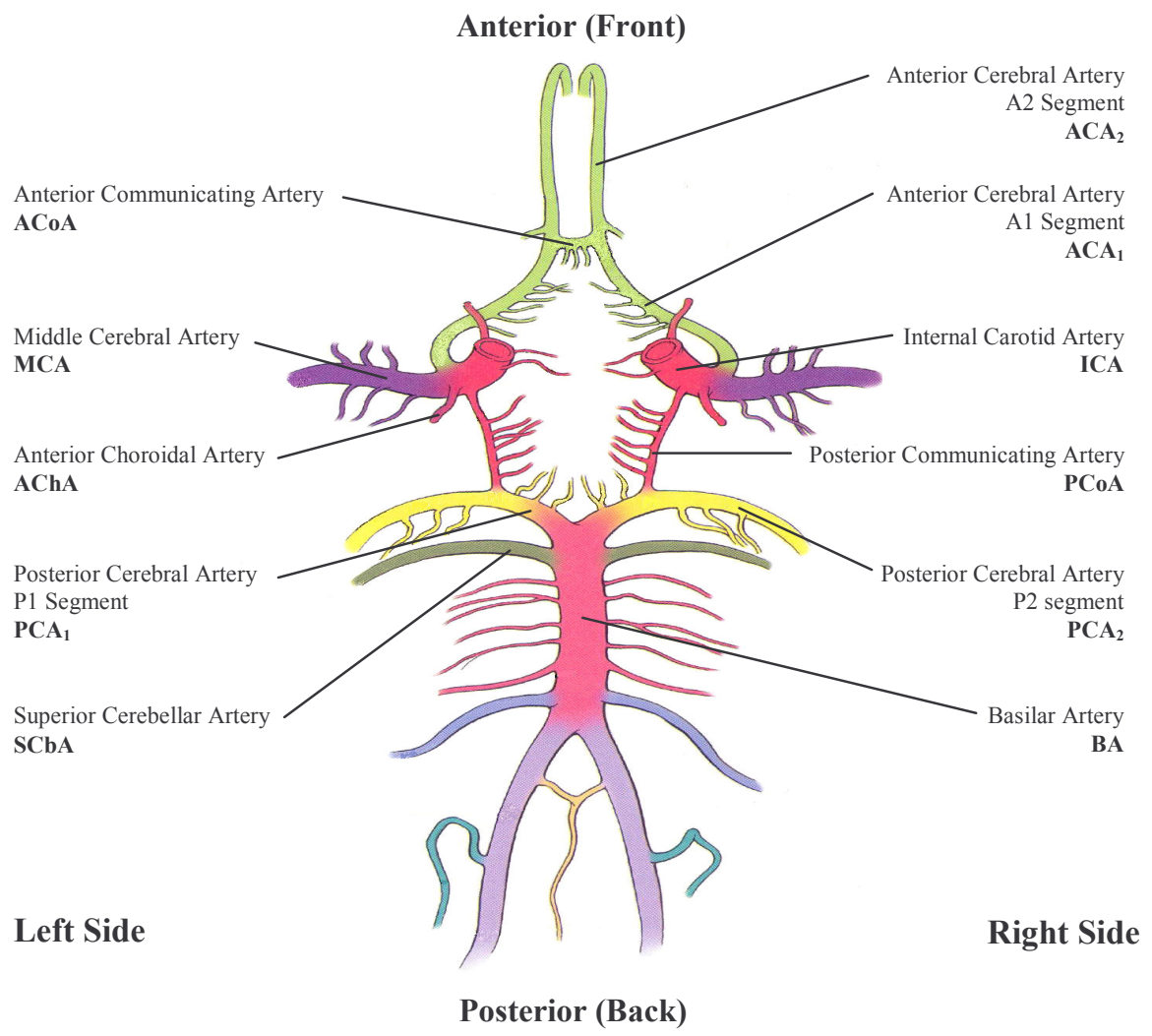

Figure 1: Circle of Willis structure [2] 
Afferent arteries supply the circulus arteries with fresh oxygen-rich arterial blood from the heart. The circulus arteries distribute oxygenated blood from the afferent arteries to the efferent arteries through the CoW. Irrespective of pressure variations in the afferent vessels, the circulus vessels redirect blood flow, thus allowing for a constant supply of blood to the cerebral mass as controlled by resistance from the efferent vessels. More specifically, vasoconstriction and vasodilation of smooth muscle cells surrounding arterioles downstream of the CoW modify efferent resistance thus regulating flow. Each efferent artery predominantly supplies a particular cerebral volume and there is no significant redundancy in that supply, although there is some collateral supply across the brain at the level of the arterioles. Because collateral flow is low and variable [3], it is neglected in this study, and therefore control is effectively decentralised, such that each of the territories regulates its supply independently of the requirements of the other territories.

Metabolic autoregulation is a means of maintaining a constant supply of nutrients to the brain despite changes in metabolism, blood oxygen content, or the rate of blood flow. The vasodilator theory on which the current model is based, suggests that when there is a decrease in oxygen delivery to the tissues or an increase in metabolism, there is a greater rate of formation of a vasodilator substance. Many different vasodilator substances have been hypothesised, such as adenosine, hydrogen ions, carbon dioxide, lactic acid, phosphate compounds, prostaglandins and potassium ions, the most important of which appear to be adenosine and hydrogen ions $[4,5]$. This vasodilator substance diffuses to the smooth muscle cells where it interferes with the $\mathrm{Ca}^{2+}$ dynamics associated with muscle contraction. Therefore, each efferent territory can independently regulate resistance and thus flow. 
While many individuals have a complete CoW geometry, it is not uncommon for some arteries to be restricted or omitted [6]. Of the circulus vessels, the communicating vessels have a higher occurrence of omission [7-9]. Physiological data has shown that although the circle can be symmetric in configuration, it is very common for the actual dimensions, and hence resistance values, to vary from the left to the right side in the $\mathrm{ACA}_{1}, \mathrm{PCoA}$ and $\mathrm{PCA}_{1}$ arteries. While an individual possessing one of these variations may under normal circumstances suffer no ill effects, there are certain clinical conditions, which compounded with effects of an absent or altered vessel, can lead to an ischaemic stroke.

To perfectly model the full distribution of vessels throughout the cerebral mass, every vessel out to the smallest arterioles would be incorporated in the model. The CoW supplies a large network of interconnected vessels, which branch from the efferent vessels. In this study, the vessels supplying cerebral tissue have been reduced to the ten main efferent vessels, due to lack of physiological data, as well as for geometric simplicity and reduced computation. Each of the ten efferent vessels in the model contains a time varying peripheral resistance to encompass the effects of the arterioles and capillaries and their autoregulation function; essentially a 'lumped parameter' model.

Ideal distribution of blood depends largely on the anatomical structure of the CoW being complete and in the same configuration shown in Figure 1. This configuration is referred to as the balanced configuration. However, it should be noted that only approximately $20 \%$ of dysfunctional brains [8] and $50 \%$ of normal brains [10] exhibit a balanced configuration. Many different types of abnormalities have been observed, 
such as absent vessels, fused vessels, string-like vessels and accessory vessels [7]. This study is limited to the investigation of the effects of absent and string-like vessels.

Prior research has resulted in a variety of computational solutions [11-15]. Most of these studies either neglect autoregulation, or ignore the clinically important transient dynamics that this research focuses on. It is these transient dynamics and their interaction with autoregulation that can lead to ischaemic outcomes.

The current research employs a one-dimensional CFD model to capture the dynamics of cerebral blood flow and the autoregulation phenomenon. Autoregulation is modelled using PID feedback controllers to modify efferent artery resistances and partial pressures of oxygen to maintain optimal efferent flowrates for a given circle geometry and afferent blood pressure. This model is computationally much faster than higher order CFD models, while retaining a high level of accuracy. Hence, the clinical relevance of this model is in its use to identify stroke-risk patients prior to surgery or other clinical procedures.

\section{Theory}

\subsection{Geometry}

The geometry required to develop the 1D model was based on a Magnetic Resonance Angiogram (MRA) of an individual's cerebro-vasculature. Artery lengths were taken from the MRA data, and artery diameters were taken from Hillen [16], representing 
an average for the human population. Hence a patient specific model could be created from similar MRA data.

Figure 2 shows the basic schematic representation of the CoW model, including efferent and afferent arteries. A sign convention for flow around the circle is indicated. Afferent and circulus arteries are shown with constant resistances, as it is assumed that smooth muscle cell induced constriction and dilation of the artery walls does not occur to a significant degree in large, relatively rigid cerebral arteries [17]. The model uses the Poiseuille flow approximation whereby the resistance of an arterial segment is inversely proportional to the fourth power of the radius of the vessel. Therefore, small changes in the radius of small peripheral arteries will cause the greatest changes in resistance, and thus flowrate. Efferent arteries are consequently shown with time-varying resistances that represent the combined resistance to flow of the smaller arteries and arterioles downstream of the CoW.

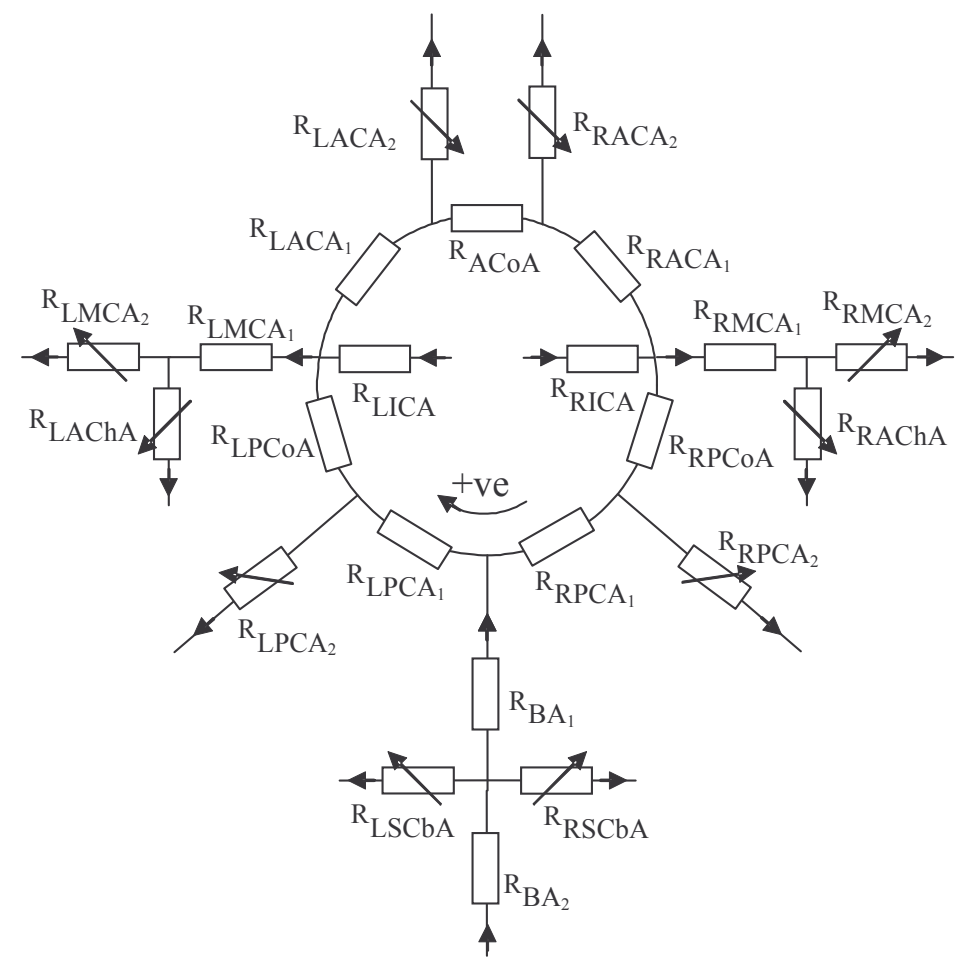

Figure 2: Schematic representation of the Circle of Willis 
The autoregulation mechanism responds to perturbations of the efferent flows from their reference value, as these perturbations lead to changes in oxygen supply to the tissue. To obtain realistic models of the flow, the evaluation of these reference fluxes is critically important. Hillen [11] suggested a total influx into the Circle of Willis of $12.5 \mathrm{~cm}^{3} \mathrm{~s}^{-1}$, and a peripheral resistance ratio of $6: 3: 4$ for the ACA, MCA and PCA's respectively. For the smaller AChA and SCbA arteries, less is known and Hillen [11] did not include these arteries. Therefore, a resistance ratio of 75:75 is defined, which produces similar pressures on the upstream side of the porous blocks as the other major efferent arteries and contributes less than 5\% of the total efferent flux, [18].

\subsection{Fluid Model}

The CoW is essentially modelled as a one-dimensional structure with laminar, viscous and incompressible flow. Per the simplifying assumption made by Ferrandez et al. [15] and Moorhead et al. [19], the flow in any arterial element is assumed to be Newtonian and axi-symmetric. Therefore, it can be modelled by the Poiseuille Equation for flow in a tube, where the flowrate, $Q$, is a function of the pressure gradient along the vessel, $\Delta P$, and the resistance, $R$, to blood flow; and the resistance is a function of the artery length, $l$, artery radius, $r$, and dynamic viscosity of blood, $\mu$ :

$$
\begin{aligned}
& Q=\frac{\Delta P}{R} \\
& R=\frac{8 \mu l}{\pi r^{4}}
\end{aligned}
$$


The assumption of Poiseuille flow implies a straight blood vessel and a fully developed velocity profile are present. In reality, these assumptions may not hold for smaller circulus vessels such as the ACoA where the vessel may be too short for a velocity profile to fully develop, and the PCoA's which take complex paths through space, resulting in an underestimated resistance value. Furthermore, the diameters of a number of the arterial segments comprising the circle are not of constant diameter, especially at junctions, resulting in some energy losses which are not represented in this model.

Equations (1) and (2) are applied to each arterial element and combined with equations for the conservation of mass at each vessel junction into a matrix equation:

$$
\underline{A} \widetilde{x}=\widetilde{b}
$$

where $\underline{A}$ is a matrix containing resistances for each arterial element, $\widetilde{x}$ is a state vector containing flowrates through those arteries and nodal pressures at the end of the arteries such that $\tilde{x}(t)=\left\{q_{1} \ldots q 23, P_{1} \ldots P_{10}\right\}^{T}$, and $\widetilde{b}$ is a vector containing arterial and venous boundary pressures. Note that if the afferent pressures in $\tilde{b}$, which drive the system, change dynamically, a time-varying system is created. The state vector $\tilde{x}$, then also becomes time-dependent, as the solution varies with each deviation in afferent pressure.

$$
\underline{A} \widetilde{x}(t)=\tilde{b}(t)
$$


The system in Equation (4) more accurately represents typical behaviour, as these afferent mean arterial input pressures are not typically constant.

\subsection{Autoregulation Dynamics}

This model represents a metabolic model of autoregulation, whereby a decrease in blood flowrate results in a decreased interstitial oxygen partial pressure, causing a greater formation of vasodilator substances diffusing to the smooth muscle cells to cause muscle relaxation. The result is a change in efferent resistance. Thus there are two dynamics: 1) for oxygen signal pathway dynamics, and 2) for the resulting smooth muscle contraction/dilation dynamics.

Guyton and Hall [20] (see Figure 3), showed that autoregulation is efficient between mean arterial pressures of approximately $70 \mathrm{mmHg}$ and $170 \mathrm{mmHg}$, but is ineffective at pressures outside this range, indicating a limit to the autoregulatory system. Normal cerebral blood flow is $48-50 \mathrm{~mL}$ blood $/ 100 \mathrm{~g}$ cerebral tissue $/ \mathrm{min}$. However, if this reference flow is not obtained, stroke will not necessarily ensue automatically. The ischaemic threshold is generally considered to be approximately $20 \mathrm{~mL} / 100 \mathrm{~g} / \mathrm{min}$, although it should be noted that tissue perfused at $20 \mathrm{~mL} / 100 \mathrm{~g} / \mathrm{min}$ is not necessarily irreversibly damaged immediately, since the length of time that tissues are perfused at such levels plays an important role [21, 22]. For the purposes of this study, perfusion of $20 \mathrm{~mL} / 100 \mathrm{~g} / \mathrm{min}$ or lower is assumed to cause stoke. As such, a 'stroke line' is inserted onto this Guyton curve as shown in Figure 3. 


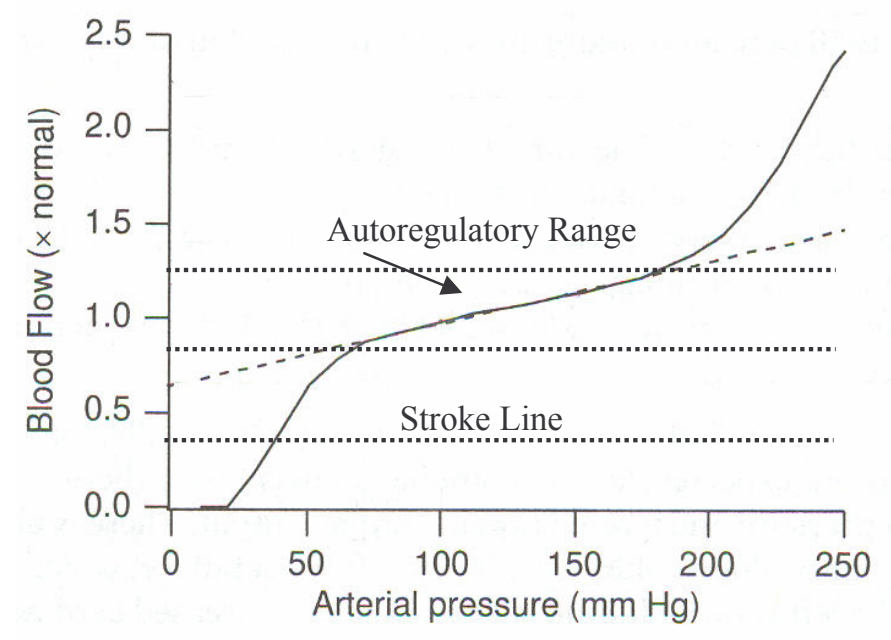

Figure 3: Effect of mean arterial pressure on blood flow

The plateau region of Figure 3 can be modelled by Equation (5), where flowrate, $Q$, is expressed as a function of arterial oxygen concentration, $\left[\mathrm{O}_{2}\right]_{a}$, metabolic rate, $M$, and arterial and venous pressures, $P_{a}$ and $P_{v},[23]$.

$$
Q=\frac{1}{1+(B)\left[O_{2}\right]_{a}}\left((M)(B)+\frac{P_{a}-P_{v}}{C}\right)
$$

Here $B$ indicates the sensitivity of resistance to oxygen, and $C$ is a parameter linking arterial resistance with venous oxygen content. Both of these parameters can be found for the steady state using normal values for arterial and venous oxygen concentration of $104 \mathrm{mmHg}$ and $40 \mathrm{mmHg}$ respectively, as well as a normal metabolic rate defined by Equation (6):

$$
M=Q\left(\left[\mathrm{O}_{2}\right]_{a}-\left[\mathrm{O}_{2}\right]_{v}\right)
$$

Figure 4 shows that Equation (5) accurately follows the Guyton model up to pressures of $220 \mathrm{mmHg}$. 


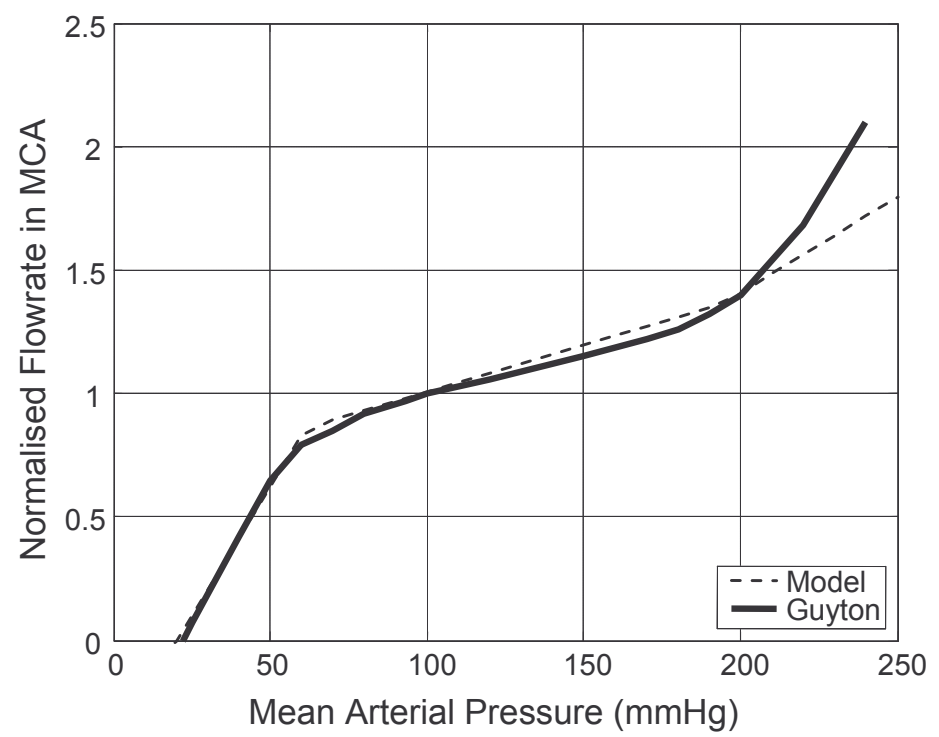

Figure 4: Flowrate as a function of arterial pressure

Efferent arteries are modelled with variable resistors capable of variations in resistance of between $50 \%$ and $155 \%$

$$
0.5 R_{\text {ref }}<R<1.55 R_{\text {ref }}
$$

Note that these variations are consistent with Figure 3, but are slightly tighter than the clinical observations of Fog et al. [24], who found a maximum dilation corresponding to a $57 \%$ decrease in resistance. However, clinical data is found to be widely varying $[25]$.

Outside the central autoregulatory range, autoregulation is unable to restore flowrates to their reference values. Hence, they rise above or fall below the desirable range of flow, resulting in stroke at the extreme of MAP. Reference flowrates are therefore set to follow the plateau region in Figure 4, even if it is not physically possible to 
achieve. This approach captures the body's drive to maintain a desired range of flow at any near arterial pressure.

When arterial blood reaches the peripheral tissues, the pressure difference encountered drives the diffusion of oxygen from the blood to the tissues. If the blood flow through a particular tissue increases, there is a greater transport of oxygen such that the oxygen concentration in the interstitial fluid increases above the normal 40 mmHg. The rate of oxygen consumption (ie metabolic rate) can also play a role here, although only normal metabolic rates are considered at this time.

A curve has been fitted to the data of Guyton and Hall [20] for oxygen partial pressure, $i \mathrm{PO}_{2}$, versus flowrate, $Q$, as shown in Figure 5, such that:

$$
i P O_{2}=(Q+64)\left(1-e^{(-0.87 Q)}\right)
$$

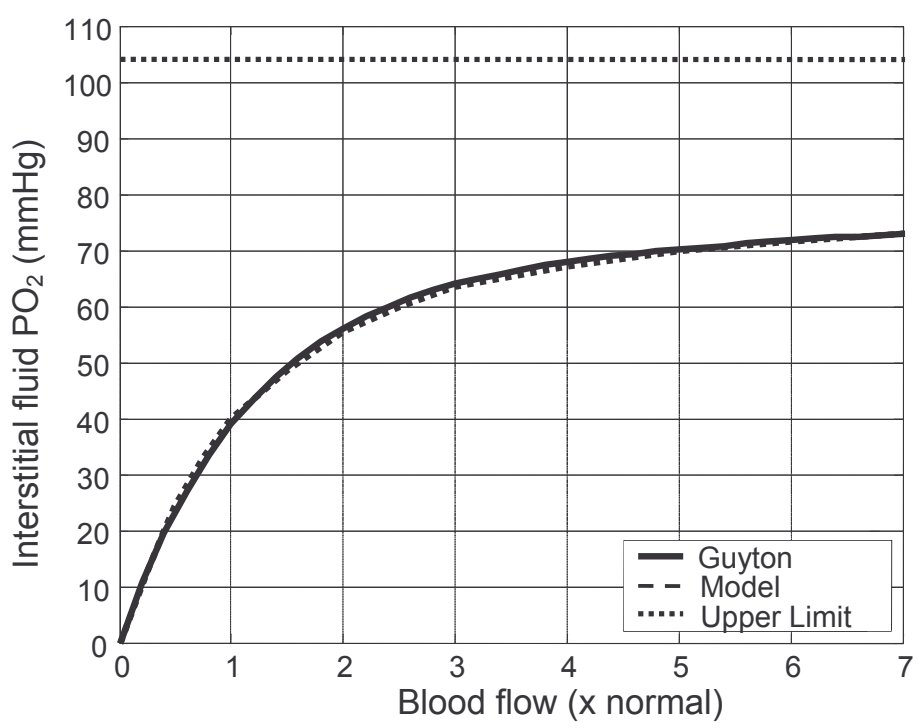

Figure 5: Interstitial oxygen partial pressure as a function of flowrate 
The autoregulation process can now be generically described by relatively simple feedback control systems, where the control input, $u(t)$, is a function of the error from a reference value, $e$, at that timestep:

$$
u(t)=K_{p} e+K_{i} \int e d t+K_{d} \frac{d e}{d t}
$$

where $K_{p}, K_{i}$ and $K_{d}$ represent proportional, integral and derivative control gains respectively, and are found by matching clinical data from Newell et al. [26], in which thigh cuff experiments were performed and the percentage change and duration of the time-dependent velocity profiles were measured using Transcranial Doppler (TCD) recordings of the MCA. As observed in Figure 6, releasing cuff pressure resulted in a $20 \%$ drop in blood pressure, where the MCA took approximately 20 seconds to return to normal flow conditions.

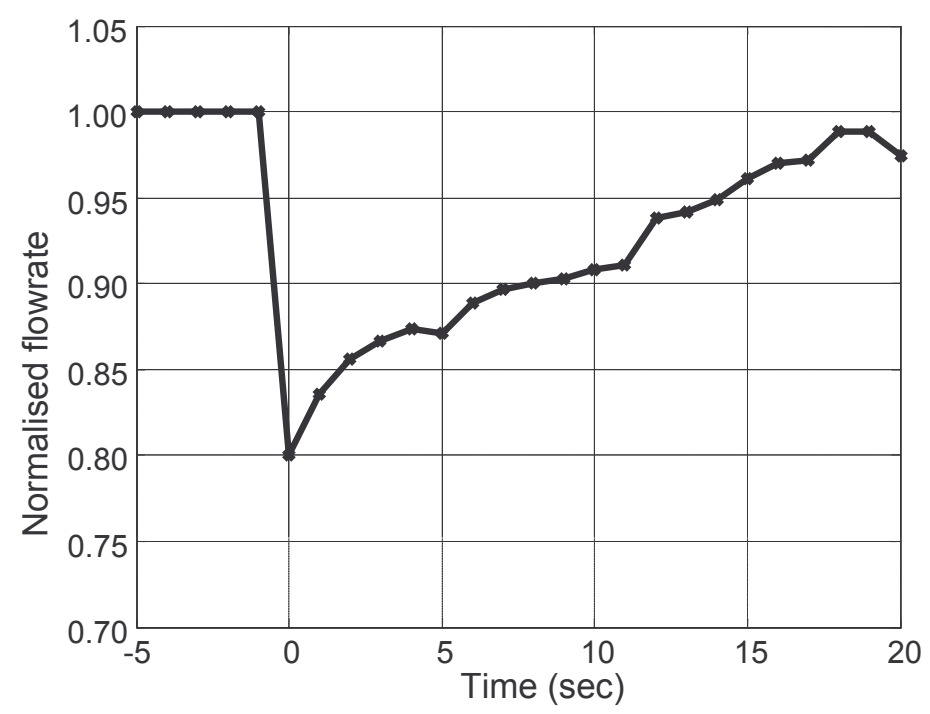

Figure 6: MCA flux profile after 20mmHg afferent pressure drop

The dynamic behaviour of the system can be described by simple first order systems of the form: 


$$
\dot{x}=\frac{1}{\tau_{1}}\left(x_{r e f}-x\right)+\frac{1}{\tau_{2}} u(t)
$$

where $x$ is the parameter in question (partial pressure of oxygen or peripheral arterial resistance), $\tau_{1}$ and $\tau_{2}$ are time constants associated with the biochemical pathways, and $u(t)$ is defined in Equation (9).

Based on the pressure at each timestep, the reference flowrate can be found using equations (5), (6) and (7), and therefore the correct oxygen partial pressure can be found from equation (8). Inner iterations use the error in flowrate and in oxygen partial pressure to calculate the control input required to correct the error. The solution process is shown in Figure 7. 


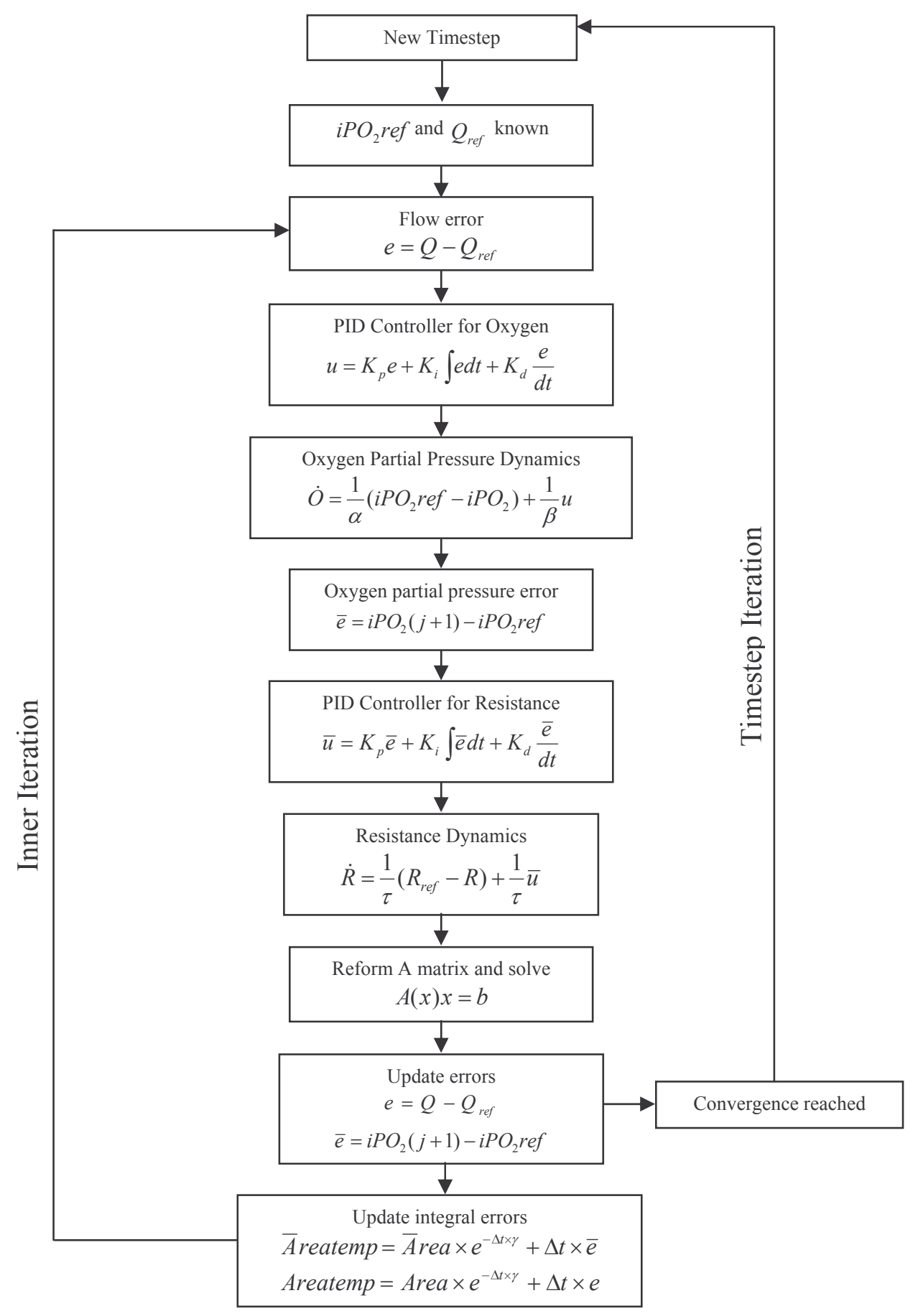

Figure 7: Solution method

At each timestep, the afferent pressures are known. Therefore, so are reference values, $Q_{\text {ref }}$ and $i P O_{2 r e f}$. When there is a change in afferent pressure, the flowrate, $Q$, calculated using resistances from the previous timestep, varies from $Q_{r e f}$. Using the 
algorithm shown in Figure 7 the new flow error is then re-calculated as indicated in "update errors" and the inner iteration continues until convergence and thus equilibrium is reached.

The time constant associated with resistance dynamics is $3 \mathrm{sec},(\tau=3$ in Figure 7$)$ whereas the time constant associated with oxygen dynamics is $35 \mathrm{sec},(\alpha=35$ in Figure 7). These values indicate that constriction/dilation of vessels in response to changes in the concentration of vasodilator substances occurs faster than oxygen delivery to the tissues and the accumulation/reduction of metabolites in response to changes in flowrate, as expected. These time constants were found by matching the velocity profiles of Newell et al. [26], and it should be noted that Ferrandez et al. [15] also used $\tau=3$ in their model.

For the oxygen dynamics, the integral gain is the major control component, whereas for the resistance dynamics, the derivative gain is the most important. Integral memory has been incorporated into the model to ensure a graded error, and it explains how metabolites are only active in the bloodstream for a finite period of time.

\section{Model Verification and Simulation Methods}

The overall system model allows for many variations in circle geometry. To simulate an absent vessel, the resistance of that vessel is set to a large value ensuring negligible flow through that segment. Similarly, simulation of string-like vessels can be performed by setting resistances to as much as sixteen times their original value. An increase in resistance of sixteen times is equivalent to a decrease in artery radius of 
$50 \%$. In addition, any type of time-varying afferent mean arterial pressure profile is easily simulated. These simulations have been used to model rapid pressure drops caused by surgical procedures, such as a carotid endarterectomy. Several combinations of clinically verified geometries and situations can be modelled using far less computational effort than similar models using higher dimensional CFD.

\subsection{Model Verification using Clinical Data}

Newell et al. [26] performed thigh cuff experiments in which releasing cuff pressure caused a pressure drop in all afferent CoW vessels from $100 \mathrm{mmHg}$, dropping to approximately $80 \mathrm{mmHg}$ before climbing to $86.2 \mathrm{mmHg}$, as shown in Figure 8 .

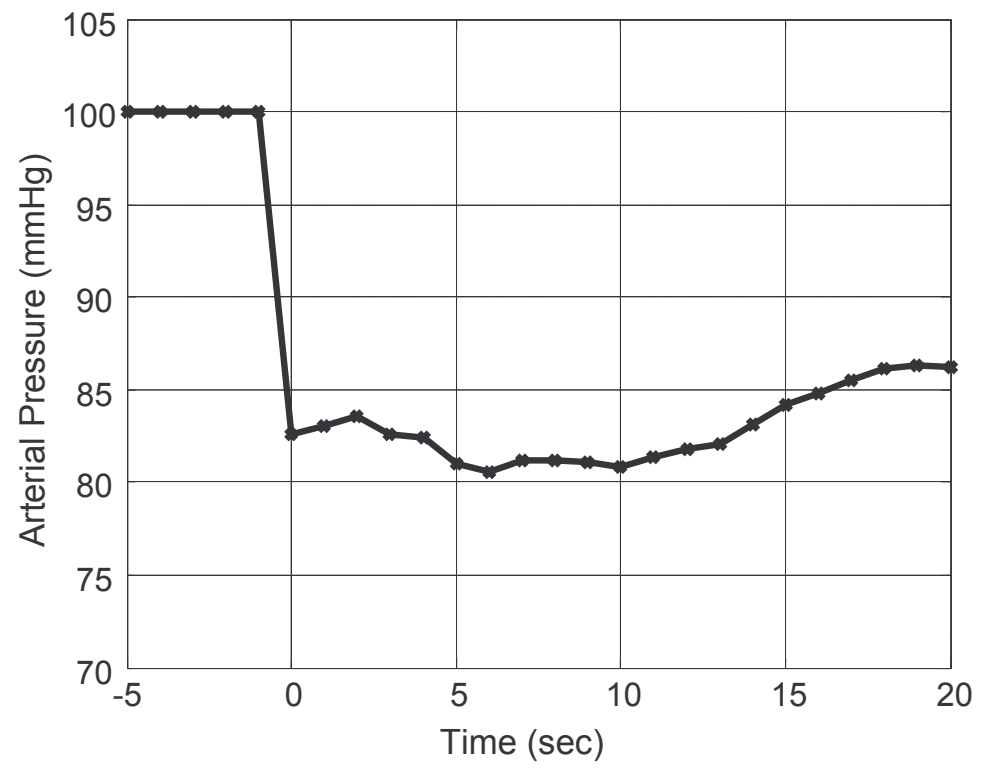

Figure 8: Newell pressure data

The complete CoW is modelled with venous pressures of $7 \mathrm{mmHg}$, intracranial pressure of $10 \mathrm{mmHg}$ [27], and an arterial pressure profile following that of Newell et al. [26] in all afferent arteries. Figure 9 shows a comparison between the current metabolic model and the results of Newell et al. [26]. 


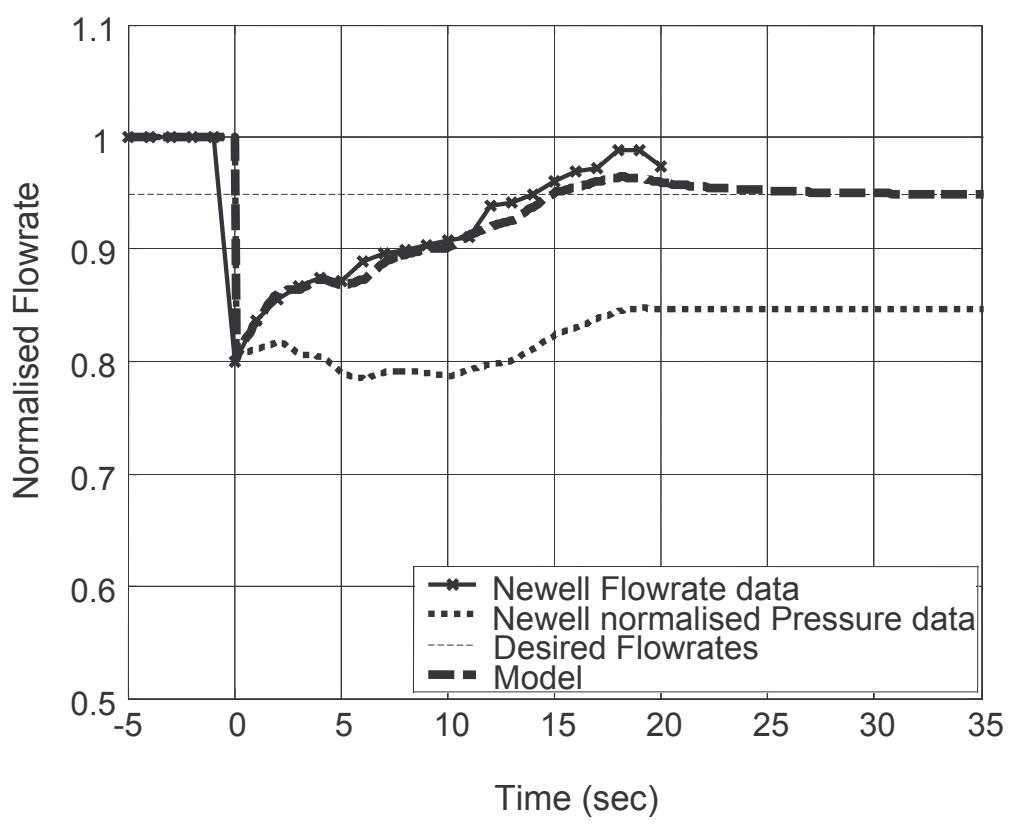

Figure 9: Comparison of current metabolic model with Newell flux profile

Note that the maximum error in Figure 9 is $2.58 \%$, while the average error is $1.03 \%$, thus the model shows excellent correlation with this clinical result.

\subsection{Accounting for Asymmetric Afferent Arterial Pressures}

Figure 9 resulted from modelling the same pressure drop in all afferent arteries. To model a stenosis or occlusion in one afferent artery, the input pressures will vary in each afferent artery. Figure 4 is used to find the desired flowrates in this case. To model a $20 \mathrm{mmHg}$ pressure drop in the RICA, the model of Figure 4 is run without a pressure drop with afferent pressures of $100 \mathrm{mmHg}$ in the LICA and BA, and $80 \mathrm{mmHg}$ in the RICA. The steady state efferent flowrates obtained are the reference flowrates to be used in the main metabolic autoregulation model, during a transient analysis of a RICA drop of $100 \mathrm{mmHg}$ to $80 \mathrm{mmHg}$. 
Simulations are carried out for the balanced configuration and each case where a single circulus vessel is omitted. In all cases where only one vessel in the CoW was removed, the system is able to obtain the reference efferent flowrates upon simulation of a surgical procedure causing a temporal pressure drop of approximately $20 \%$ in the RICA. To reach the reference flowrates the flow is redirected as a result of independently changing resistance in the vascular bed of each efferent artery - the autoregulation function.

\section{Results and Discussion}

The results displayed in the remainder of this research use a bar chart format. The $\mathrm{x}$ axis has afferent vessels on the left, with flowrates through efferent arteries shown on the right. Bars below the $\mathrm{x}$-axis indicate that flow is in the opposite direction to that assumed by the sign convention in Figure 2. The four bars indicate from left to right: 1) flowrates before the pressure drop, 2) flowrates after recovery from the pressure drop, 3) the desired reference flowrates after the pressure drop, and 4) the ischaemic threshold. The goal of autoregulation is to match the second and third bars. Schematic figures show flow directions after the pressure drop, where solid arrows indicate a change in direction of flow from the steady state, pre-pressure drop case, and open arrows indicate that the flow direction has not changed.

\subsection{Balanced Configuration}

The 'normal' case with a complete CoW is used as a baseline for analysing results. The solid black bars in Figure 10 therefore show zero flowrate through the ACoA and equal flowrates across each of the left and right sections of the circle. For this case the 
BA supplies mainly the efferent PCA's, and the ICA's supply the efferent MCA's and ACA's. These results are in good agreement with the accepted physiological response [26].

A sudden occlusion in the RICA is simulated by a pressure drop from 100 to 80 $\mathrm{mmHg}$, with the resulting flowrates shown in Figure 10. When compared to the normal pre-pressure drop scenario, flow is observed to drop in the RICA and increase in the LICA and BA to compensate. The pressure change in the RICA also causes a change in the direction of flow through the RACA 1 resulting in a clockwise flow through the anterior regions of the circle, delivering flow from the LICA through the ACoA to the starved $\mathrm{RACA}_{2}$ and RMCA. Lastly, note that all efferent arteries recover reference flowrates after the pressure drop.

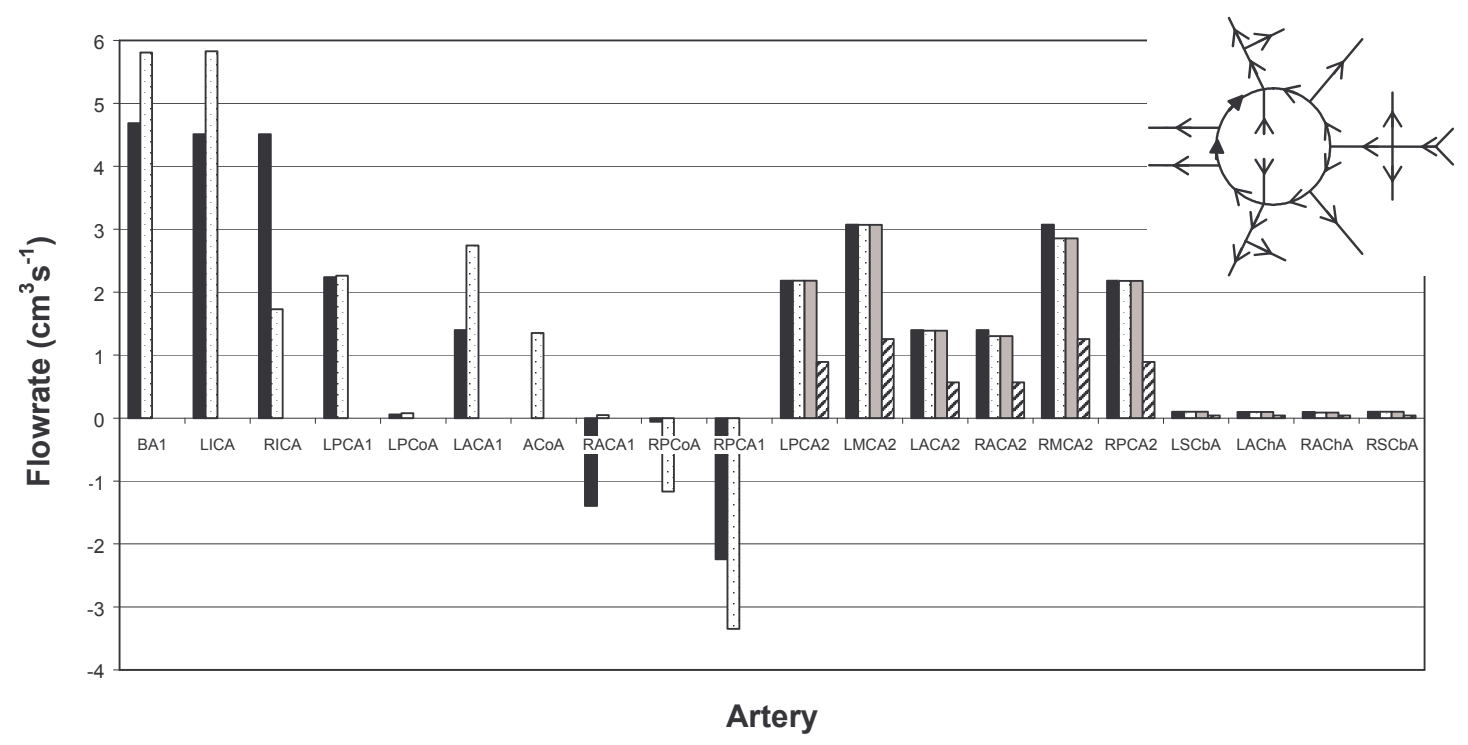

Before Stenosis $\square$ After Stenosis $\square$ Desired Efferent Flowrates after Stenosis $\square$ Stroke Line

Figure 10: Balanced configuration response to $20 \mathrm{mmHg}$ pressure drop in RICA 
The step change in pressure from $100 \mathrm{mmHg}$ to $80 \mathrm{mmHg}$ in the RICA, results in significant changes in the flowrate and the pressure differential in the RMCA. Specifically, the flowrate for both RMCA and $\mathrm{RACA}_{2}$ dropped $20 \%$ and took approximately 20 seconds to return to their original value, as shown in Figure 11. Flowrates in Figure 11 are normalised to the RMCA pre-pressure drop reference flowrate, whilst the SCbA's and AChA's are not shown due to the negligible amount of flow through these vessels. However, as seen in Figure 10, they do obtain their reference values following the pressure drop.
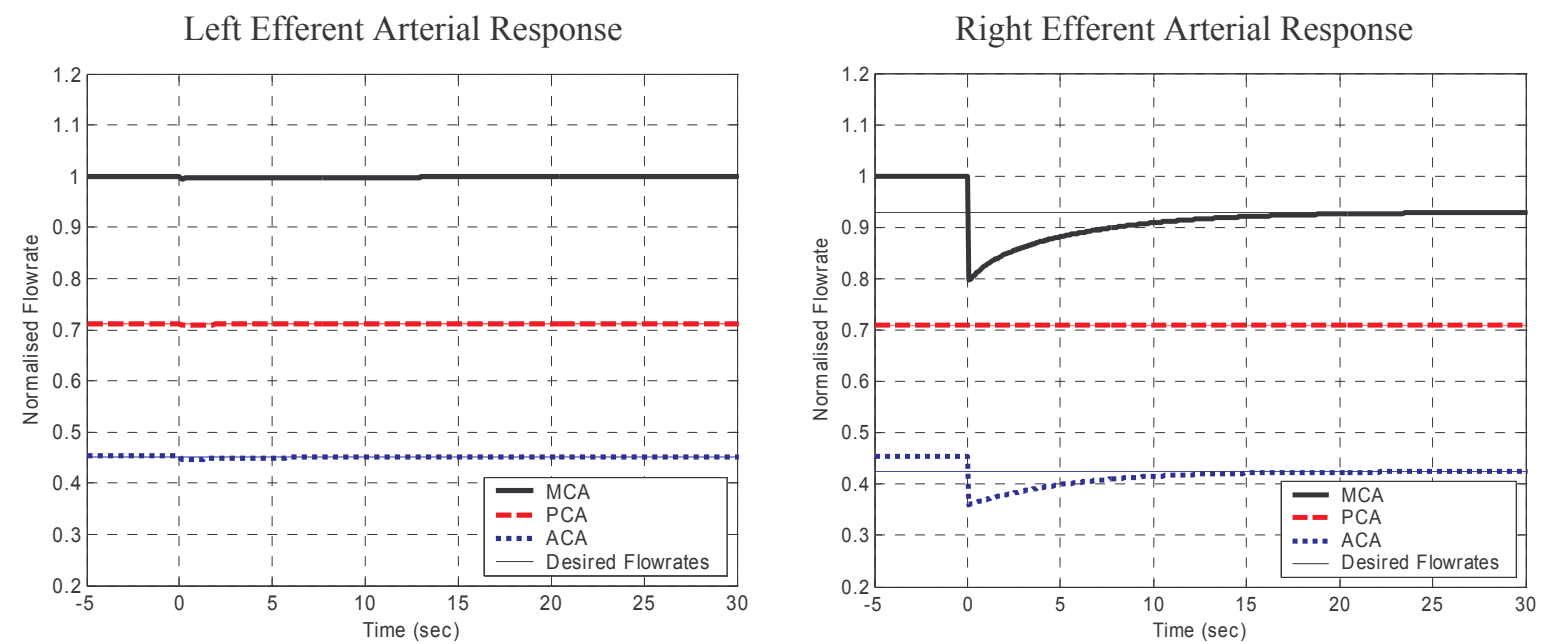

Figure 11: Efferent arterial response to $20 \mathrm{mmHg}$ pressure drop in RICA - Balanced configuration

\subsection{Absent ACoA}

An absent or restricted ACoA is a common physiological condition [10]. Under normal conditions an absent ACoA poses no threat to brain function as shown by the zero flowrate through the ACoA in the normal balanced configuration case in Figure 10. The complication of the omission of the ACoA is only significant under flow conditions such as a stenosis or occlusion of an afferent artery, where other arteries are absent, or where there is asymmetry in the circle. 
Unlike the balanced case, the simulation for the absent ACoA case results in little change from the normal flow case. The reason for the low change is that the ACoA can no longer be used to reroute flow from the LICA to help supply the starved right efferent vessels. Flow in the left portion of the brain is virtually unaffected by the reduced flow in the RICA. Flow through the $\mathrm{RACA}_{1}$ changes only to account for the new target $\mathrm{ACA}_{2}$ flowrate after the pressure drop. Immediately after the RICA pressure drop, flow increases through the LMCA and $\mathrm{LACA}_{2}$ by $0.2 \%$ and $0.3 \%$, respectively. This result is due to an increase in flow through the LICA immediately after the pressure drop to try to compensate for the loss in the RICA. After the LMCA is supplied, the extra flow must go to the $\mathrm{LACA}_{2}$ since no flow can pass through the ACoA. Flow through the LICA then decreases, since its previous increase was not helping supply the right efferent vessels. Flowrates through the LMCA and $\mathrm{LACA}_{2}$ then decrease to their reference values.

Before the pressure drop, flow through the left and right $\mathrm{PCA}_{1}$ were identical, whereas afterwards, flow through the RPCA $A_{1}$ increased significantly. This is a result of the BA delivering blood at a higher rate through the RPCA 1 and RPCoA to supply the RMCA. Thus the communicating arteries play a more significant role when the geometry is "unbalanced", as seen in the flowrate changes in Figure 12. 


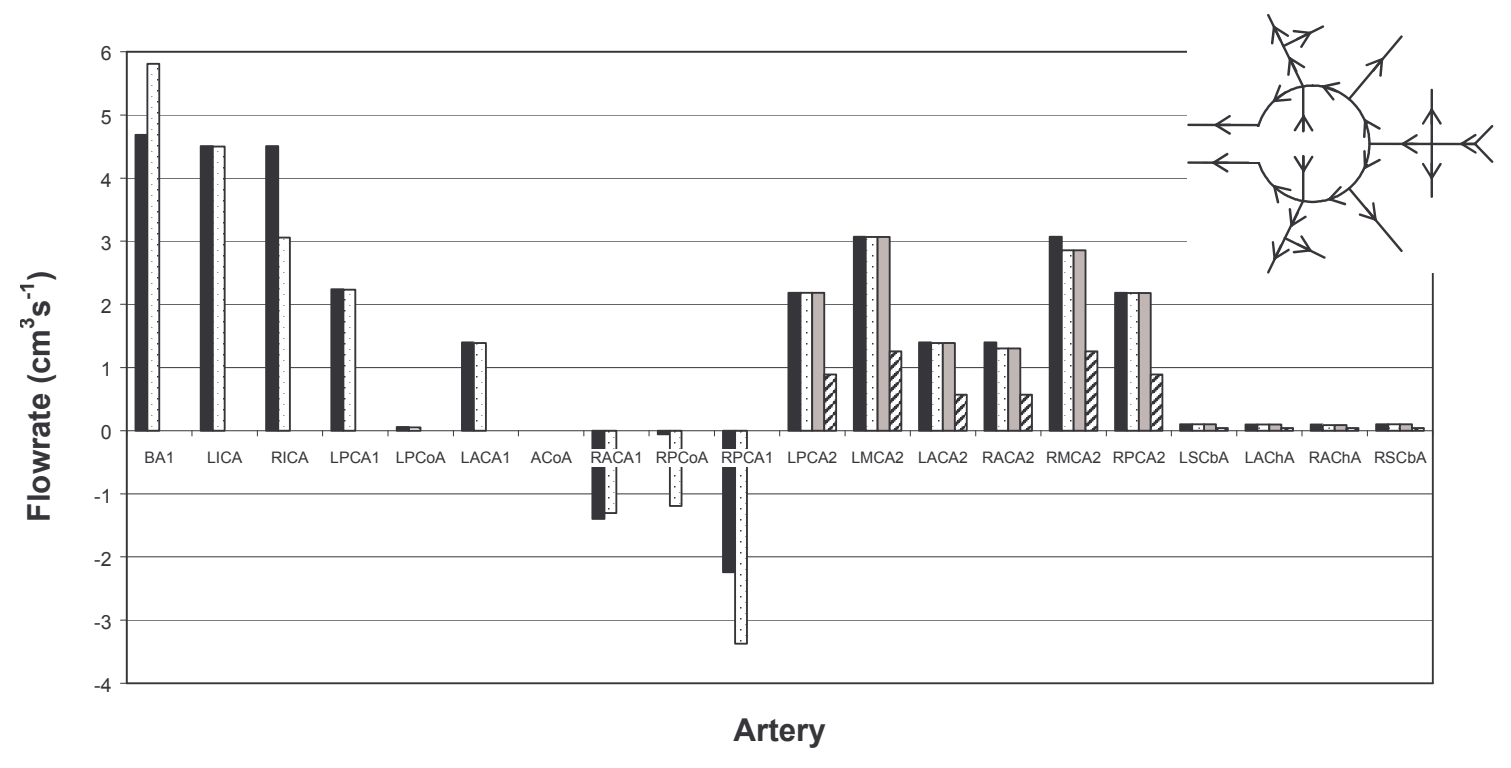

Before Stenosis $\square$ After Stenosis $\square$ Desired Efferent Flowrates after Stenosis $\square$ Stroke Line

Figure 12: Absent ACoA configuration response to $20 \mathrm{mmHg}$ pressure drop in RICA

\subsection{Absent RACA or $_{1} \mathrm{LACA}_{1}$}

This case was very similar to an absent ACoA response in that there was very little difference between the pre-pressure drop scenario and the stenosis situation, especially in the left portion of the brain. In both the absent RACA 1 and $\mathrm{LACA}_{1}$ prepressure drop cases, the flowrate through the ACoA is much higher than any other case and over five times higher than the next highest ACoA flowrate.

For all the cases where the CoW geometry is missing a single vessel, the absent $\mathrm{LACA}_{1}$ case showed the largest immediate drop of $24 \%$ in flowrate through the $\mathrm{LACA}_{2}$ when the pressure drop was simulated. Flow through the RACA $A_{2}$ was also significantly affected, but returned to the desired flowrate.

Immediately after an RICA pressure drop in the absent $\mathrm{RACA}_{1}$ case, flow increased through the LMCA, $\mathrm{LACA}_{2}$ and $\mathrm{RACA}_{2}$ by $0.15 \%, 0.2 \%$, and $3 \%$ respectively. This 
result is due to an increase in flow through the LICA to try to compensate for the loss in the RICA.

\subsection{Absent LPCoA or RPCoA}

Missing either of the PCoA's has little effect in the pre-pressure drop case due to their high resistances. The response is very similar to the balanced case with the most significant change after the pressure drop being the flow through the ACoA. An absent RPCoA does not increase the BA flow, as in the balanced case and absent LPCoA case, since an increased BA flow cannot compensate for the reduced RICA flow with the RPCoA vessel missing. In both LPCoA and RPCoA cases, the direction of flow changes in the $\mathrm{RACA}_{1}$, as flow is redirected from the left side of the circle clockwise to the right. Immediately following the pressure drop, the RMCA is most affected in both the absent left and right PCoA cases, as it is in the balanced case, with the left and right PCA's the least affected.

\subsection{Absent RPCA or $_{1} \mathrm{LCA}_{1}$}

With the RPCA 1 missing, the BA cannot supply the RPCA 2 as it would in all other cases. Consequently, the flowrate through the BA is over $28 \%$ less than the other omitted artery cases already discussed in both the normal flow and stenosis situations. There is a much larger response to the RICA pressure drop in the RPCA 2 than in any other case with an initial reduction in flowrate of $24 \%$. The flowrate was twice as high through the RPCoA as any of the other absentee cases. After the pressure drop, the $\mathrm{RPCA}_{2}$ is only able to obtain $77 \%$ of its reference flow, although remains well above the 'stroke line'. This result is rarely clinically seen since individuals with a string-like or absent $\mathrm{PCA}_{1}$ tend to have an ipsilateral $\mathrm{PCoA}$ with an increased radius 
and/or decreased length. When this simulation is repeated with the RPCoA's length decreased by $86 \%$, and its radius increased by $136 \%$ [28], such that its overall resistance is decreased by $75 \%$, reference flow is obtained after the pressure drop.

Similarly, the BA flowrate is much lower in the absent $\mathrm{LPCA}_{1}$ case than in any other cases. The flowrate demanded from the LICA was highest in this situation demanding a $40 \%$ increase in flowrate through the LICA compared to the normal flow case. The LICA supplies the LMCA and $\mathrm{LPCA}_{2}$, and the BA supplies the $\mathrm{RPCA}_{2}$ and RMCA. In the normal flow case the $\mathrm{LACA}_{2}$ is supplied by the LICA, with a small amount of help from the RICA. With the reduction in flow in the RICA due to the pressure drop, the RACA 2 obtains its demands from the LICA, and there is only a very small amount of flow through the RACA .

\subsection{Stroke Risk Case}

All the prior cases with a single omitted artery were able to maintain the reference flowrate in all the efferent arteries, showing the robustness of the CoW system as a whole. The case below represents a realistic situation of increased stroke risk where the autoregulation process cannot keep the efferent flowrates at the desired level. A situation is modelled in which the RPCoA is absent and a sudden $80 \%$ carotid occlusion is simulated by suddenly increasing left and right ICA resistances. The RMCA, RACA 2 and RAChA drop below the ischaemic threshold representing a potential stroke, as shown in Figure 13. 


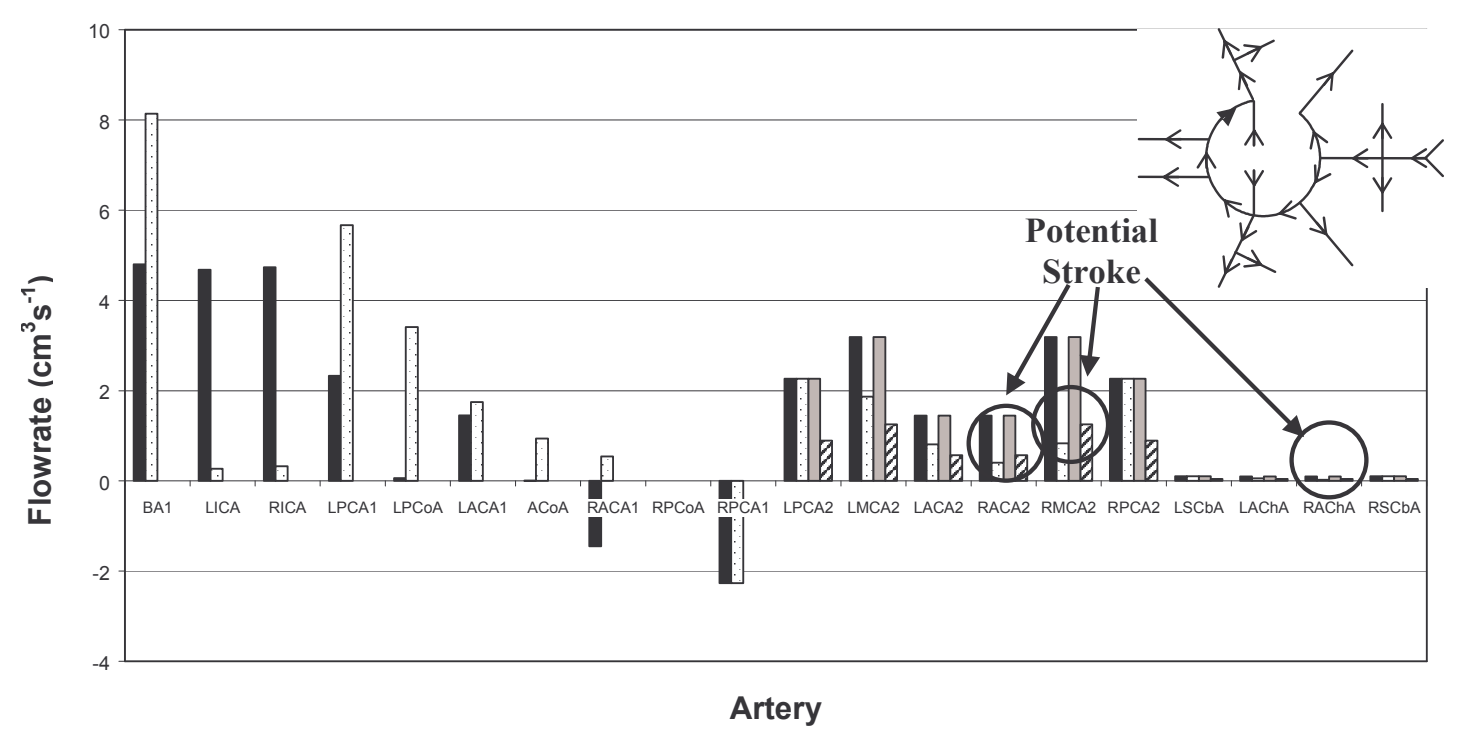

Before Stenosis $\square$ After Stenosis $\square$ Desired Efferent Flowrate after Stenosis $\square$ Stroke Line

Figure 13: 80\% carotid stenosis with absent RPCoA

This failure to return to reference flowrate occurs because the resistance of the $\mathrm{RACA}_{2}, \mathrm{RMCA}$ and RAChA cannot be further decreased since the maximum radius of these arteries has been reached as a result of the autoregulation process model. The final flowrate is not only lower than the desired value, but also falls below the ischaemic threshold, leading to reduced perfusion and higher stroke risk for this type of input. Figure 14 shows the efferent transient response. Note that flowrates in Figure 14 are normalised to each efferent artery's 'normal' balanced configuration reference value, and the SCbA's and AChA's are not shown due to the tiny amount of flow through these vessels. 


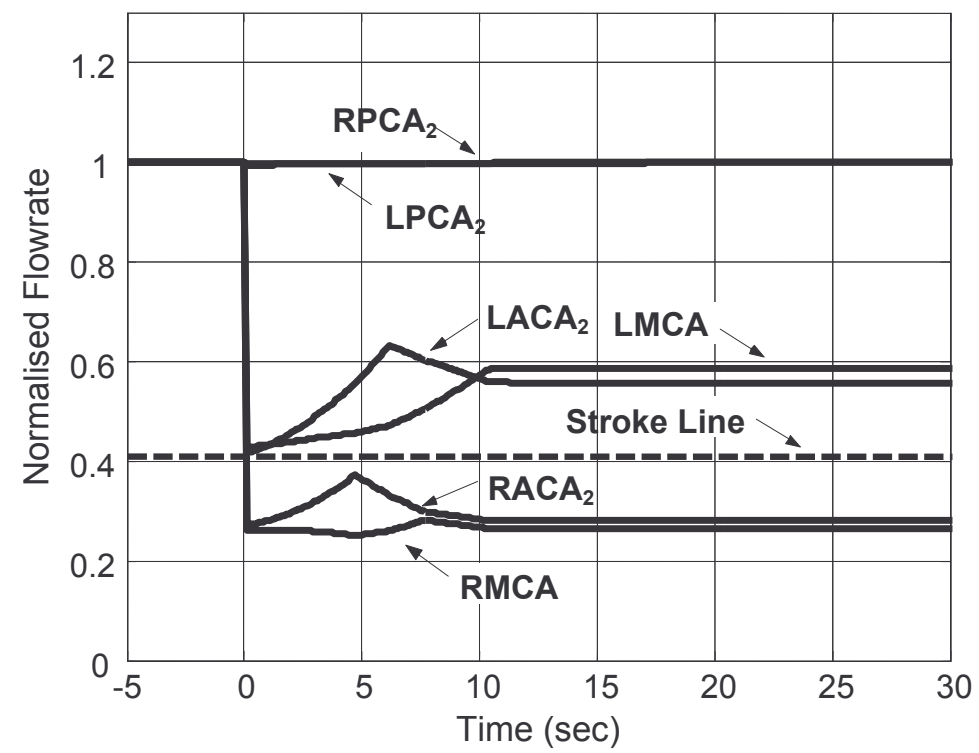

Figure 14: Efferent arterial response to $80 \%$ carotid stenosis with an absent RPCoA 


\section{Conclusions}

A one-dimensional CFD metabolic model of the Circle of Willis has been created to study autoregulation of cerebral blood flow for clinical events such as occlusions or stenoses in afferent arteries, absent or string-like circle vessels, or arterial infarctions. The model captures cerebral haemodynamic autoregulation using ProportionalIntegral-Derivative (PID) controllers to modify efferent artery resistances and partial pressures of oxygen to maintain optimal efferent flowrates for a given circle geometry and afferent blood pressure.

The model is physiologically relevant, matching the accepted physiological responses of blood flow as a function of arterial pressure, tissue oxygen partial pressure as a function of blood flow, as well as limited clinical data. The two dynamics account for metabolic autoregulation with appropriate time constants associated with firstly oxygen diffusion and metabolite generation, and secondly peripheral arterial wall vasoconstriction and vasodilation. The model is a significant advancement over preexisting models in its incorporation of inner iterations at each solution time-step to find the equilibrium state, within physiological limitations. In addition, the solution for the CoW arterial system is obtained in a far shorter time period using this timevarying resistance model than with higher dimensional CFD methods, and requires significantly less computational effort while retaining a high level of accuracy.

Results show excellent correlation with the clinical data. The time-dependent velocity profiles in the MCA were matched to give a $20 \%$ decrease in flowrate, followed by 
approximately 20 seconds to return to steady state conditions in response to a stenosis in the RICA.

Simulations have been performed with the omission of any single circulus artery and found that no such omission leads to failure in reaching the required efferent flowrates for a RICA stenosis, thus highlighting the known robustness of the CoW system. A physiologically realistic case with an absent RPCoA and a sudden $80 \%$ carotid occlusion was developed and simulated, and it was observed that the RMCA, RACA2, and RAChA flowrates fell below the ischaemic threshold, indicating a possible stroke. This result shows the effectiveness of this model as a tool for determining potential outcomes of surgical or other therapies.

Future research will aim to add models for myogenic autoregulation, in which vasodilation and vasoconstriction of peripheral vessel walls occurs, to a certain degree, directly in response to changes in transmural pressure in the artery wall. In addition, more clinical verification is required, which will also involve the modelling of a greater variety of potential $\mathrm{CoW}$ geometries using clinical data. Correlation to retrospective outcomes will indicate any specific model limitations and increase the level of validation. 


\section{References}

[1] Tortora, G. (1997). "Introduction to the Human Body. The Essentials of Anatomy and Physiology", $4^{\text {th }}$ Edition. Chapter 10, pp 228. Addison Wesley Longman, Inc, USA.

[2] Vander, A., Sherman, J., Luciano, D. (2001). "Human Physiology - The Mechanisms of Body Function”, McGraw Hill, Eighth Edition.

[3] Brozici, M., van der Zwan, A., Hillen, B. (2003). "Anatomy and Functionality of Leptomeningeal Anastomoses A Review”, Stroke 34:2750-2762.

[4] Klabunde, R. (2004). "Cardiovascular Physiology Concepts", Lippincott Williams \& Wilkins, USA. (http://www.cvphysiology.com/).

[5] Guyton, A.C. (1996). "Textbook of Medical Physiology", $8^{\text {th }}$ Edition. W.B. Saunders Company, USA. Chapter 17, pp 200-205.

[6] van der Zwan, A. and Hillen, B. (1991). "Review of the variability of the territories of the major cerebral arteries", Stroke 22(8): 1078-84.

[7] Alpers, B. and R. Berry (1963). "Circle of Willis in Cerebral Vascular Disorders, The Anatomical Structure", Archives of Neurology 8: 398-402.

[8] Riggs, H. and C. Rupp (1963). "Variation in Form of Circle of Willis. The Relation of the Variations to Collateral Circulation: Anatomic Analysis", Archives of Neurology 8: 24-30.

[9] Battacharji, S., E. Hutchinson, et al. (1967). "The Circle of Willis - The Incidence of Developmental Abnormalities in Normal and Infarcted Brains", Brain 90: 747-758.

[10] Alpers, B., R. Berry, et al. (1959). "Anatomical Studies of the Circle of Willis in Normal Brain", AMA Archives of Neurology 81: 409-418.

[11] Hillen, B., H. Hoogstraten, et al. (1986). "A Mathematical Model of the Flow in the Circle of Willis", Journal of Biomechanics 19(3): 187-194.

[12] Cassot, F., Zagzoule, M., Marc-Vergnes, J. (2000). "Hemodynamic role of the circle of Willis in stenoses of internal carotid arteries. An analytical solution of a linear model", Journal of Biomechanics 33 (2000) 395-405.

[13] Lodi, C. A., Ursino, M. (1999). "Hemodynamic Effect of Cerebral Vasospasm in Humans: A Modeling Study", Annals of Biomedical Engineering vol. 27: 257-273. 
[14] Hudetz, A. G., Halsey, J. H. Jnr., Horton, C. H., Conger, K. A., Reneau, D. D. (1982). "Mathematical Simulation of Cerebral Blood Flow in Focal Ischemia", Stroke 13(5): 693-700.

[15] Ferrandez, A., T. David, et al. (2002). "Numerical models of auto-regulation and blood flow in the cerebral circulation", Computer Methods in Biomechanics and Biomedical Engineering 5(1): 7-19.

[16] Hillen, B., A. Drinkenburg, et al. (1988). "Analysis of Flow and Vascular Resistance in a Model of the Circle of Willis", Journal of Biomechanics 21(10): 807-814.

[17] Fung, Y. C. (1990). "Biomechanics: Motion, Flow, Stress and Growth", Springer-Verlag Inc., New York.

[18] Moore, S, Moorhead, KT, Chase, JG, David, T and Fink, J (2005). "1D and 3D Models of Cerebrovascular Flow”, ASME J. Biomechanics, volume 127, pp 440-449.

[19] Moorhead, K T, Doran, C V, Chase, J G and David, T (2004). "Lumped Parameter and Feedback Control Models of the Auto-Regulatory Response in the Circle of Willis", Computer Methods in Biomechanics and Biomedical Engineering, volume 7 (3), pp 121-130, ISSN: 1025-5842 .

[20] Guyton, A.C. (1996). “Textbook of Medical Physiology”, $8^{\text {th }}$ Edition. W.B. Saunders Company, USA.

[21] Heiss, WD, Thiel, A, Grond, M, and Graf, R (1999). "Which targets are relevant for therapy of acute ischemic stroke?" Stroke 1999;30:1486 -1489.

[22] Baron JC (2001). "Perfusion thresholds in human cerebral ischemia: historical perspective and therapeutic implications", Cerebrovasc Dis. 2001;11(suppl 1):2-8.

[23] Keener, J, Sneyd, J (1998). "Mathematical Physiology”, Springer-Verlag, New York, Inc.

[24] Fog, M (1937). "The reaction pial arteries to a fall in blood pressure", Arch. Neurol. Pyschiat. 37:351-364.

[25] Dirnagl, U, Pulsinelli, W (1999). "Autoregulation of Cerebral Blood Flow in Experimental Focal Brain Iscemia", Journal of Cerebral Blood Flow and Metabolism, 10:327-336.

[26] Newell, D., R. Aaslid, et al. (1994). "Comparison of Flow and Velocity During Dynamic Autoregulation Testing in Humans", Stroke 25: 793-797.

[27] Walters, FJM (1998). "Intracranial Pressure and Cerebral Blood Flow", Update in Anaesthesia, Issue 8:Article 4:1-4. 
[28] Arnold, J, Fink, J, David, T, Chase, JG, Moore, S, Moorhead, KT and Alzaidi, S (2004). "Anatomical variations of the Circle of Willis and magnetic resonance measurement techniques", Proc. of the 2004 Annual Conference of the Australasian College of Physical Scientists and Engineers in Medicine (ACPEM), Christchurch, New Zealand, Nov. 22-23. 